\title{
Biphasisches Mesotheliom bei einer Braunviehkuh: Klinische, histomorphologische, immunhistochemische und elektronenmikroskopische Befunde
}

\author{
Braun, Ueli ; Rütten, Maja ; Bleul, Ulrich ; Previtali, Matteo ; Krüger, S ; Gerspach, Christian ; Geiger, \\ $\mathrm{S}$; Sydler, Titus
}

\begin{abstract}
Im vorliegenden Fallbericht wird eine 10-jährige hochträchtige Braunviehkuh mit dem auffälligsten äusseren Befund eines stark dilatierten Abdomens mit konturlosem Übergang vom Euter zur ventralen Bauchwand vorgestellt. Die sonographische Untersuchung ergab einen hochgradigen Aszites und mehrere echogene knotige Verdickungen im grossen Netz. Aufgrund sämtlicher Befunde wurde die Diagnose nichtentzündlicher Aszites, vermutlich aufgrund des Tumors gestellt. Die Kuh wurde infolge infauster Prognose nach erfolgreich eingeleiteter Geburt euthanasiert und seziert. Bei der Eröffnung der Bauchhöhle flossen 248.5 Liter klare Aszitesflüssigkeit ab. Das grosse Netz war sulzig verdickt und teilweise mit unterschiedlich grossen, zystischen und flüssigkeitsgefüllten Hohlräumen durchsetzt. Aufgrund der histologischen, immunhistochemischen und elektronenmikroskopischen Befunde wurde die Diagnose biphasisches Mesotheliom mit Zystenbildung im gesamten Bauchraum gestellt. Abstract:A 10-year-old Swiss Braunvieh cow near term was referred to our clinic because of severe abdominal distension, which caused loss of demarcation between the udder and ventral abdominal wall. Ultrasonographic examination revealed marked ascites and multiple echogenic nodules in the greater omentum. Based on the findings, non-inflammatory ascites attributable to neoplasia was diagnosed. Rupture of the prepubic tendon from the pubic symphysis was also suspected. Because of a grave prognosis, parturition was induced and a live calf was delivered. The cow was euthanized and a postmortem examination was carried out. The abdominal cavity contained 248.5 litres of clear fluid. The greater omentum was thickened and oedematous and regionally contained fluid-filled cystic structures, which varied in size with a maximum diameter of 10 centimetres. Based on the histological, immunohistochemical and electron microscopical findings, biphasic mesothelioma with cyst formation affecting the entire abdominal cavity was diagnosed.
\end{abstract}

DOI: https://doi.org/10.1024/0036-7281/a000290

Other titles: Biphasic mesothelioma in a Swiss Braunvieh cow: Clinical, histological, immunohistochemical and electron microscopical findings

Posted at the Zurich Open Repository and Archive, University of Zurich

ZORA URL: https://doi.org/10.5167/uzh-55048

Journal Article

Accepted Version

Originally published at:

Braun, Ueli; Rütten, Maja; Bleul, Ulrich; Previtali, Matteo; Krüger, S; Gerspach, Christian; Geiger, S; Sydler, Titus (2012). Biphasisches Mesotheliom bei einer Braunviehkuh: Klinische, histomorphologis- 
che, immunhistochemische und elektronenmikroskopische Befunde. Schweizer Archiv für Tierheilkunde, 154(1):33-38

DOI: https://doi.org/10.1024/0036-7281/a000290 
1 Biphasisches Mesotheliom bei einer Braunviehkuh: Klinische, histomorphologische,

2 immunhistochemische und elektronenmikroskopische Befunde

4 U. Braun ${ }^{1}$, M. Rütten ${ }^{2}$, U. Bleul ${ }^{1}$, M. Previtali ${ }^{1}$, S. Krüger ${ }^{1}$, C. Gerspach ${ }^{1}$, S. Geiger ${ }^{2}$, T. Sydler ${ }^{2}$

${ }^{1}$ Departement für Nutztiere und ${ }^{2}$ Institut für Veterinärpathologie der Universität Zürich

\section{Zusammenfassung}

9 Im vorliegenden Fallbericht wird eine 10-jährige hochträchtige Braunviehkuh mit dem auffälligsten äusseren Befund eines stark dilatiertens Abdomens mit konturlosem Übergang vom Euter zur

11 ventralen Bauchwand vorgestellt. Die sonographische Untersuchung ergab einen hochgradigen

12 Aszites und zudem mehrere echogene knotige Verdickungen im grossen Netz. Aufgrund sämtlicher

13 Befunde wurde die Diagnose nichtentzündlicher Aszites, vermutlich aufgrund des Tumors gestellt.

14 Die Kuh wurde infolge infauster Prognose nach erfolgreich eingeleiteter Geburt euthanasiert und 15 seziert. Bei der Eröffnung der Bauchhöhle flossen 248.5 Liter klare Aszitesflüssigkeit ab. Das grosse

16 Netz war sulzig verdickt und teilweise mit unterschiedlich grossen, zystischen und

17 flüssigkeitsgefüllten Hohlräumen durchsetzt. Aufgrund der histologischen, immunhistochemischen

18 und elektronenmikroskopischen Befunde wurde die Diagnose biphasisches Mesotheliom mit

19 Zystenbildung im gesamten Bauchraum gestellt.

21 Schlüsselwörter: Rind, Aszites, biphasisches Mesotheliom, Abriss Bauchwandmuskulatur

Biphasic mesothelioma in a Swiss Braunvieh cow: Clinical, histological, immunohistochemical and electron microscopical findings

A 10-year-old Swiss Braunvieh cow near term was referred to our clinic because of severe abdominal distension, which caused loss of demarcation between the udder and ventral abdominal wall. Ultrasonographic examination revealed marked ascites and multiple echogenic nodules in the greater omentum. Based on the findings, non-inflammatory ascites attributable to neoplasia was diagnosed. Rupture of the prepubic tendon from the pubic symphysis was also suspected. Because of a grave prognosis, parturition was induced and a live calf was delivered. The cow was euthanased and a postmortem examination was carried out. The abdominal cavity contained 248.5 litres of clear fluid.

The greater omentum was thickened and oedematous and regionally contained fluid-filled cystic 
structures, which varied in size with a maximum diameter of 10 centimetres. Based on the

35 histological, immunohistochemical and electron microscopical findings, biphasic mesothelioma with cyst formation affecting the entire abdominal cavity was diagnosed.

Keywords: cattle, ascites, biphasic mesothelioma, prepubic tendon rupture

\section{Einleitung}

41 Mesotheliome sind seltene Tumoren und in der Regel bösartig. Sie kommen am häufigsten beim adulten Rind vor und gehen von Pleura, Perikard, Peritoneum oder Tunica vaginalis aus (Brown et al., 2007). Beim Kalb wurden kongenitale, bei 1- bis 2-jährigen Rindern kongenitale und erworbene

Formen beschrieben. Beim Rind sind Mesotheliome in der Regel vom epitheloiden Typ und kommen primär im Peritoneum vor (Schulmann, 2003). Sie gehen von der Serosa oder vom subserosalen Bindegewebe aus und können beide Lokalisationen gleichzeitig betreffen. Bei den Wiederkäuern ist in erster Linie das Rind (Wolfe et al., 1991; Pizarro et al., 1992; Renner und Ruager, 1993; Tammen et al., 1994; Girard, 1995; Beytut, 2002; Braun et al., 2004; Takasu et al., 2006) von dieser Neoplasie betroffen; gelegentlich kommt sie jedoch auch bei der Ziege (McCullagh et al., 1979; Krametter et al., 2004; Braun et al., 2009) und nur äusserst selten beim Schaf (Brown und Weaver, 1981) vor. Kühe mit einem Mesotheliom des Peritoneums weisen als wichtigste Befunde eine Umfangsvermehrung des Abdomens auf, welche durch einen hochgradigen Aszites verursacht wird. In seltenen Fällen können die Tumorknoten auch rektal palpiert werden (Braun et al., 2004). Die sonographische Untersuchung ist für die Abklärung hilfreich. Diese ergibt als Hauptbefunde eine massive Ansammlung von echoarmer Flüssigkeit im Abdomen und/oder Thorax (Wolfe et al., 1991;

Milne et al., 2001; Braun et al., 2004). Zudem sind die tumorösen Veränderungen als echogene knotige Bezirke auf dem Netz, den Serosen und Organoberflächen wie zum Beispiel Pansen und Psalter zu sehen. Das Bauchhöhlenpunktat weist die Charakteristika eines modifizierten Transsudats auf. Die zytologische Diagnose eines Mesothelioms kann sich schwierig gestalten, da die Punktate häufig zellarm sind. Zudem stellen sich entartete und reaktive Mesothelzellen morphologisch ähnlich dar und sind manchmal nur schwer zu unterscheiden. Die Verdachtsdiagnose eines Mesothelioms wird bei der Ultraschalluntersuchung aufgrund der mit Aszites einhergehenden knotigen Veränderungen im Abdomen gestellt. Differentialdiagnostisch müssen andere Tumoren wie Karzinome und Sarkome, Tuberkulose in den Serosen (Perlsucht) und Fettgewebsnekrosen in Betracht gezogen werden (Brown et al., 2007). Die endgültige Diagnose erfolgt aufgrund der histologischen, immunhistochemischen oder elektronenmikroskopischen Untersuchung der tumorösen Veränderungen. Die vorliegende Arbeit beschreibt ein biphasisches Mesotheliom, welches 
aufgrund unserer Kenntnis bisher bei keiner Tierart beschrieben wurde und auch nicht der Einteilung der WHO (Schulmann, 2003) entspricht.

\section{Anamnese, klinische Befunde, Laborbefunde}

72 Die erkrankte Kuh gehörte der Schweizer Braunviehrasse an. Sie war 10 Jahre alt und 9 Monate 10

73 Tage trächtig. Seit ca. 4 Wochen hatte der Besitzer eine übermässige Umfangsvermehrung des Abdomens beobachtet. Er stellte die Kuh deshalb zuerst seinem Tierarzt vor. Von diesem wurde sie wegen unklaren Befunden zur Untersuchung in die Klinik überwiesen. Der auffälligste Befund war ein massiv dilatiertes Abdomen mit konturlosem Übergang vom Euter zur ventralen Bauchwand (Abb. 1). Das Allgemeinbefinden war mittelgradig gestört, der Hautturgor war reduziert und die Skleralgefässe waren injiziert. Die Herzfrequenz betrug 84 Schläge pro Minute, die Atemfrequenz 44 Atemzüge pro Minute und die rektale Temperatur $39.4^{\circ} \mathrm{C}$. Die Pansenmotorik war aufgehoben und die Pansenfüllung war reduziert. Von den Fremdkörperschmerzproben erwies sich der Rückengriff als positiv. Die Schwing- und Perkussionsauskultation waren beidseits negativ. Darmmotorik war vorhanden und die Bauchdecke war gespannt. Bei der Rektaluntersuchung war eine fortgeschrittene Gravidität mit lebendem Kalb feststellbar. Kotfarbe, -konsistenz und -menge waren normal. Der Harn war makroskopisch und bei der Untersuchung mit dem Streifentest (Combur ${ }^{9}$-Test, Roche, Basel) unauffällig. Das Gleiche gilt für die hämatologischen Befunde und die Aktivitäten der Leberenzyme. Von den Elektrolyten war die anorganische Phosphatkonzentration mit $0.6 \mathrm{mmol} / \mathrm{l}$ erniedrigt (normal 1.3 - $2.4 \mathrm{mmol} / \mathrm{l}$ ). Die Harnstoffkonzentration war mit $6.4 \mathrm{mmol} / \mathrm{l}$ normal (normal $2.4-6.5 \mathrm{mmol} / \mathrm{l}$ ) und die Kreatininkonzentrationen mit $153 \mu \mathrm{mol} / \mathrm{l}$ leicht erhöht (normal 55 - 103 $\mu \mathrm{mol} / \mathrm{l})$. Das Ergebnis einer venösen Blutgasanalyse war ohne Besonderheiten und das Pansenchlorid lag mit $22 \mathrm{mmol} / \mathrm{l} \mathrm{im}$ normalen Bereich $(15-30 \mathrm{mmol} / \mathrm{l})$.

\section{Ultraschall- und Zystoskopiebefunde}

93 Bei der Ultraschalluntersuchung erwies sich das ganze Abdomen mit echoarmer Flüssigkeit gefüllt 94 und auf dem grossen Netz waren echogene knotige Verdickungen zu sehen (Abb. 2). Die Punktion

95 ergab eine goldgelbe klare Flüssigkeit mit einem spezifischen Gewicht von 1026 und einem

96 Proteingehalt von $33 \mathrm{~g} / \mathrm{l}$. Die Harnstoffkonzentration betrug $6.6 \mathrm{mmol} / \mathrm{l}$, die Kreatininkonzentration

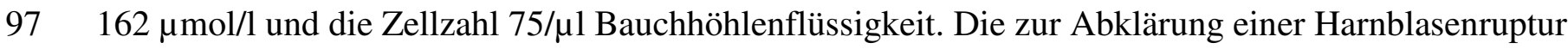
98 durchgeführte Zystoskopie war unauffällig. 
101 Aufgrund sämtlicher Befunde wurde die Diagnose nichtentzündlicher Aszites gestellt. Als Ursache 102 wurde ein Tumor vermutet. Zudem bestand Verdacht auf einen Abriss des geraden Bauchmuskels 103 von der Beckensymphyse. Da die Prognose infaust und die Kuh kurz vor dem errechneten

104 Geburtstermin war, wurde die Geburt mit 15 mg des synthetischen Prostaglandin-Analogons

105 Luprostiol (Prosolvin, Virbac, Glattbrugg) i. m. eingeleitet. Zwei Tage später kam es zur normalen

106 Geburt eines gesunden Kalbes. Die Kuh wurde danach euthanasiert und seziert. Das Gewicht des

107 Tierkörpers betrug vor der Eröffnung der Bauchhöhle $920 \mathrm{~kg}$ und nach dem Abfliessen der

108 Bauchhöhlenflüssigkeit noch 665 kg. Die Flüssigkeit wies demnach ein Gewicht von 255 kg auf, was 109 beim oben genannten spezifischen Gewicht 248.5 Litern entspricht. Die Flüssigkeit war von der 110 bereits beim Punktat beschriebenen Beschaffenheit. Die inneren Organe des Brust- und Bauchraums 111 (Lunge, Leber, Niere, Uterus) waren makroskopisch und histologisch unauffällig. Die

112 Bauchwandmuskulatur war rechts im Bereich des Ansatzes des Beckens vollständig rupturiert. Das 113 grosse Netz war stellenweise sulzig verändert und bis zu 4 cm dick. Das Netz und das Mesenterium 114 waren von zystischen Hohlräumen durchsetzt (Abb. 3), die einen Durchmesser von bis zu $10 \mathrm{~cm}$ 115 aufwiesen und eine gelb-klare Flüssigkeit enthielten. Zudem konnten im Netz und Mesenterium 116 weissliche Plaques mit einem Durchmesser von bis zu $0.5 \mathrm{~cm}$ gefunden werden. Die Innenwand der 117 zystischen und flüssigkeitsgefüllten Hohlräume war ebenfalls teilweise mit plaqueartigen bis flach118 papillären Wucherungen bedeckt. Histologisch bestanden die Zystenwände aus einem bis über $1 \mathrm{~cm}$ 119 dicken Proliferat, welches aus zwei Komponenten aufgebaut war. Die erste Komponente bestand aus 120 einem einschichtigen, epithelialen vorwiegend azinär, teilweise aber auch papillär wachsenden 121 Proliferat (Abb. 4). Die neoplastischen Epithelzellen wiesen gelegentlich feine Zilien, einen 122 mittelgradigen Gehalt von eosinophilem Zytoplasma und runde bis ovale, meist zentralliegende 123 Zellkerne mit einer vesikulären Chromatinstruktur auf. Die epitheliale Komponente wies nur eine 124 geringe Anisozytose und Anisokaryose auf. Die Mitoserate war sehr niedrig bis vernachlässigbar. Die 125 zweite Komponente bestand aus einem selbstständig proliferierenden, oft die epithelialen Strukturen 126 unterstützenden mesenchymalen Proliferat. Diese Zellen waren spindelig mit kaum erkennbaren 127 Zellgrenzen und in oft sich verflechtenden Bündeln oder Wirbeln gelegen, welche von einem feinen, 128 fibrovaskulären Stroma unterteilt waren. Die Zellkerne der neoplastischen Zellen waren plump129 spindelig bis oval mit einer feinen netzartigen Chromatinstruktur und häufig kleinen multiplen 130 Nukleolen. Die spindelige Komponente zeigte eine mittelgradige Anisozytose, Anisokaryose und 131 Anisonukleoliose. Auch hier waren nur vereinzelt Mitosefiguren erkennbar. Mit der Elastica-van132 Gieson-Färbung liessen sich die spindeligen Komponenten teilweise gelb für Muskelfasern und rot 133 für Bindegewebe anfärben. 
134 Immunhistochemisch reagierten der epitheliale Anteil und nur ganz vereinzelte langgezogene 135 spindelige Zellen mit einem Pan-Zytokeratinmarker (Cytokeratin, Klon MNF116 von Dako) stark 136 positiv (Abb. 5). Der spindelige Anteil hingegen reagierte stark positiv mit Vimentin, einem Marker

137 für mesenchymale Zellen (Dako, Klon 3 B4), alpha smooth muscle actin (Dako, Klon 1A4) und 138 geringgradig mit Desmin (Dako, Klon D33), was für eine Differenzierung der spindeligen Zellen in

139 Richtung Myofibroblasten sprach. Daneben produzierten diese Zellen in deutlichen Mengen auch 140 extrazelluläre Kollagenfasern.

141 Da die Diagnose histomorphologisch und immunhistochemisch nicht eindeutig gestellt werden 142 konnte, wurde eine elektronenmikroskopische Untersuchung durchgeführt (Lucas et al., 2003).

143 Die neoplastische Epithelzellen stellten sich als hochdifferenzierte epitheliale Zellen mit zum Lumen 144 hin ausgebildeten Zilien und Mikrovilli, gut entwickelten Desmosomen, und zum Teil überlangen 145 Desmosomen-ähnlichen Strukturen zwischen den neoplastischen Zellen dar (Abb. 6). Die epithelialen 146 Zellen wiesen grosse, etwas unregelmässig geformte Nuklei auf und sassen auf einer gut 147 ausgebildeten Basalmembran. Die spindelige neoplastische Komponente glich fibroblastischen Zellen 148 und wies auch extrazellulär kollagene Fibrillen mit der klassischen Periodizität auf.

149 Intrazytoplasmatisch wurden oft irregulär angeordnete Aktinfilamente mit zentralliegender

150 Verdichtung und Reste von rauhem endoplasmatischem Retikulum gefunden. Die Zellkerne waren 151 teilweise sehr gross und langgezogen mit gut ausgebildeten Nukleolen. In einigen der spindeligen 152 Zellen waren epitheliale Charakteristika bzw. Strukturen wie direkter Zellkontakt, Desmosomen153 ähnliche Strukturen und intrazytoplasmatische, gut ausgebildete Zilien zu sehen, was als 154 pathognostisch für tumorös entartete Zellen angesehen wird (Suzuki et al., 1976). Aufgrund all dieser 155 Befunde wurde die Diagnose biphasisches Mesotheliom mit ausgeprägter Zystenbildung gestellt.

\section{Diskussion}

158 Ein grosses Abdomen stellt für den Tierarzt immer eine diagnostische Herausforderung dar, da dafür 159 viele Ursachen in Frage kommen. Bei einer hochträchtigen Kuh, wie im vorliegenden Fall, besteht 160 immer zuerst der Verdacht, dass die Umfangsvermehrung des Bauches mit der Gravidität im 161 Zusammenhang steht. Einerseits ist an eine Zwillings- oder sogar Drillingsgravidität, andererseits an 162 eine Eihautwassersucht zu denken. Ersteres war die Vermutung des Tierbesitzers, sodass er den 163 Tierarzt erst beizog, als die Kuh kaum mehr aufstehen konnte. Letzteres, die Eihautwassersucht, 164 konnte schon vom einweisenden Tierarzt mit grosser Wahrscheinlichkeit ausgeschlossen werden, da 165 bei der rektalen Untersuchung ein Kalb palpiert wurde und der Uterus nicht übermässig gross 166 erschien. Insbesondere fehlte bei der rektalen Untersuchung die für eine Eihautwassersucht typische 167 ballonartige Ausdehnung des Uterus nach dorsal (De Kruif, 1993). Die Ultraschalluntersuchung 
168 erwies sich im vorliegenden Fall diagnostisch und prognostisch als wertvoll. Mit ihrer Hilfe konnten

169 der Aszites und echogene knotige Verdickungen im grossen Netz einwandfrei dargestellt werden.

170 Diese Befunde erlaubten die Verdachtsdiagnose einer tumorösen Erkrankung. Ein nicht entzündlicher

171 Aszites aufgrund anderer Ursachen wie Rechtsherzinsuffizienz, Vena-cava-caudalis-Thrombose oder

172 -Kompression und portale Hypertonie konnten aufgrund der klinischen und sonographischen Befunde

173 ausgeschlossen werden. Bei einer Rechtsherzinsuffizienz wären die Jugularvenen gestaut gewesen,

174 bei einer Kompression oder Obstruktion der Vena cava caudalis wäre diese auf dem Ultraschallbild

175 oval oder rund und dilatiert gewesen und bei einer portalen Hypertonie wären im Leberparenchym

176 Veränderungen zu sehen gewesen und die Portalvene wäre dilatiert gewesen. Auch ein entzündlicher

177 Aszites als Folge einer Peritonitis war aufgrund des Punktatbefunds sehr unwahrscheinlich. Die

178 vergleichende Bestimmung von Harnstoff und Kreatinin in Blut und Punktat sprach auch gegen das

179 Vorliegen eines Uroperitoneums, wie es bei Harnblasen- und/oder Urachusruptur gelegentlich

180 vorkommt (Braun et al., 2006, 2009). Zudem ergab auch die Zystoskopie keinen Hinweis für eine

181 Harnblasen- oder Urachusruptur.

182 Das ein- oder beidseitige Abreissen des geraden Bauchmuskels vom Tendo praepubicus kommt bei

183 hochträchtigen Kühen nicht selten vor (Dirksen, 2002). Es ist bei Zwillingsträchtigkeit und

184 Eihautwassersucht bekannt, während es bei Aszites unseres Wissens nach nicht beschrieben wurde.

185 Im vorliegenden Fall dürfte die Kombination von Aszites und fortgeschrittener Gravidität die

186 Ursache für den Muskelabriss gewesen sein. Die Menge von knapp 250 Litern Aszitesflüssigkeit, von

187 uns bisher nie in diesem Ausmass gesehen, stellte zusammen mit dem Kalb und den Fruchtwässern,

188 eine enorme Belastung der Muskulatur dar, was schliesslich zum Abriss führte.

189 Auffällig bei dem vorliegendem Fall war die ungewöhnliche Wachstumsart des Mesothelioms mit

190 Bildung grosser, zystenartiger Gebilde in der Bauchhöhle, welche bei der Sektion nicht sofort ins

191 Auge sprangen. Das histologische Bild des Tumors mit dem aktivem Aussehen beider Komponten

192 liess schnell den Verdacht auf ein biphasisches Mesotheliom mit zystischem Wachstum entstehen.

193 Die Schwierigkeit bestand darin, die morphologische Diagnose zu sichern. Makroskopisch und

194 histologisch konnten auch nach sorgfältiger Untersuchung keine weiteren Tumorherde in anderen

195 inneren Organen gefunden werden, welche einen anderen Ursprungsherd als die Serosa hätten

196 vermuten lassen. Mit der immunhistochemischen Untersuchung konnte bestätigt werden, dass es sich

197 um einen zusammengesetzten Tumor handelt. Als Goldstandard der Diagnosesicherung eines

198 Mesothelioms gilt auch heute noch die elektronenmikroskopische Untersuchung (Hammar, 2006).

199 Das Auffinden klarer neoplastischer Strukturen in den epithelialen Zellen und das Vorkommen von

200 mesothelialen Zellen mit eindeutigen epithelialen Komponenten liess die Diagnose biphasisches

201 Mesotheliom zu. Als wichtigste Hilfe zur Diagnosesicherung oder zum Ausschluss weiterer 
202 Differentialdiagnosen empfiehlt es sich, bei der makroskopischen Adspektion die inneren Organe 203 genau auf auch kleine Tumorherde zu untersuchen (Hammar, 2006). Makroskopisch und histologisch 204 zeigte das Mesotheliom eine ungewöhnliche Wachstumart, welche nach dem Wissen der Autoren bei 205 Tieren weder beschrieben wurde noch in der histologischen Klassifikation der WHO (Schulmann, 206 2003) vorkommt.

207 Mit dieser Arbeit soll darauf hingewiesen werden, dass eine zystische Variante des Mesothelioms 208 auch beim Tier vorkommen kann. Beim Menschen wurden multizystische peritoneale Mesotheliome 209 bereits beschrieben (Charfi et al., 2008).

\section{Literatur}

212 Beytut, E.: Metastatic sclerosing mesothelioma in a cow. Aust. Vet. J. 2002, 80: 409-411.

Braun, U., Gerspach, C., Metzger, L., Ziegler-Gohm, D.: Ultrasonographic findings in a cow with 215 ascites due to a mesothelioma. Vet. Rec. 2004, 154: 272-274.

Braun, U., Nuss, K., Wapf, P., Lischer, C.: Clinical and ultrasonographic findings in five cows with a

Braun, U., Irmer, M., Steininger, K., Schade, B.: Ultraschallbefunde bei einer Ziege mit Aszites infolge Mesotheliom. Schweiz. Arch. Tierheilk. 2009, 151: 397-400.

Braun, U., Previtali, M., Fürst, A., Wehrli, M., Muggli, E.: Zystoskopie bei einem Rind mit Urachus persistens-Ruptur. Schweiz. Arch. Tierheilk. 2009, 151: 539-544.

Brown, C. C., Baker, D. C., Barker, I. K.: Neopastic diseases of the peritoneum. In: Jubb, Kennedy, and Palmer's Pathology of Domestic Animals, Volume 2. Ed. M. Grant Maxie. Saunders Elsevier,

230 Edinburgh, London, New York, Oxford, Philadelphia, St. Louis, Sydney, Toronto, 2007, $294-296$. 
236 De Kruif, A.: Eihautwassersucht. In: Tiergeburtshilfe. Hrsg. E. Grunert, K. Arbeiter. Paul Parey

237 Verlag, Berlin, Hamburg, 1993, 142-144.

Dirksen, G.: Bauchwandbruch, Abriss des geraden Bauchmuskels. In: Innere Medizin und Chirurgie des Rindes. Hrsg. G. Dirksen, H.-D. Gründer, M. Stöber. Parey Buchverlag, Berlin, 2002, 692-695.

Hammar, S. P.: Macroscopic, histologic, histochemical, immunohistochemical, and ultrastructural features of mesothelioma. Ultrastruct. Pathol. 2006, 30: 3-17.

Krametter, R., Bagó, Z., Floeck, M., Baumgartner, W.: Abdominal mesothelioma in a goat. New Z.

Lucas, D. R., Pass, H. I., Madan, S. K., Adsay, N. V., Wali, A., Tabaczka, P., Lonardo, F.: Sarcomatoid mesothelioma and its histological mimics: a comparative immunohistochemical study. Histopathology 2003, 42: 270-279.

McGullagh, K. G., Mews, A. R., Pinsent, P. J. N.: Diffuse pleural mesothelioma in a goat. J. Vet.

Milne, M. H., Mellor, D. J., Barrett, D. C., Fitzpatrick, J. L.: Observations on ascites in nine cattle.

Pizarro, M., Brandau, C., Sanchez, M. A., Flores, J. M.: Immunocytochemical identification of a bovine peritoneal mesothelioma. J. Vet. Med. A 1992, 39: 476-480.

Renner, J. E., Ruager, J.: Mesotheliose bei einer Kuh. Dtsch. Tierärztl. Wschr. 1993, 100: 369-370.

Schulmann F. Y.: Tumors of mesothelium. In: Histological Classification of Tumors of the collaboration with the American Registry of Pathology and The World Health Organization 
269 Collaborating Center for Worldwide Reference on Comparative Oncology, Washington, 2003, 144270147.

272 Suzuki, Y., Kannerstein, M.: Utrastructure of human malignant diffuse mesothelioma. Am. J. Pathol. 273 1976, 85:241-262

275 Takasu, M., Shirota, K., Uchida, N., Iguchi, T., Nishii, N., Ohba, Y., Maeda, S., Miyazawa, K., Murase, 276 T., Kitagawa, H.: Pericardial mesothelioma in a neonatal calf. J. Vet. Med. Sci. 2006, 68: 519-521.

278 Tammen, F. C., Von Elling, U., Tammen, C., Fiedler, H. H., Stöber, M.: Schwere Geburtsbehinderung 279 beim Rind durch Bauchfell-Mesotheliose des Kalbes. Tierärztl. Prax. 1994, 22: 121-123.

281 Wolfe, D. F., Carson, R. L., Hudson, R. S., Boosinger, T. R., Mysinger, P. W., Powe, T. A., Claxton, 282 M. S., Angel, K. L.: Mesothelioma in cattle: Eight cases (1970-1988). J. Amer. Vet. Med. Assoc. 283 1991, 199: 486-491.

286 Korrespondenz

287 Ueli Braun

288 Departement für Nutztiere

289 Winterthurerstrasse 260

290 CH-8057 Zürich

291 Fax: +41 (0)44 6358904

292 E-mail: ubraun@vetclinics.uzh.ch 
293 Legende zu den Abbildungen

294 Abbildung 1: Hochträchtige, 10-jährige Braunviehkuh mit Mesotheliose und Abriss des geraden

295 Bauchmuskels. Das Abdomen ist infolge Aszites massiv dilatiert. Der konturlose Übergang vom

296 Euter zur ventralen Bauchwand ist typisch für den Muskelabriss.

298 Abbildung 2: Ultrasonographische Befunde bei einer Braunviehkuh mit Aszites infolge Mesotheliose.

299 In und ausserhalb des Netzbeutels ist eine massive Flüssigkeitsansammlung zu sehen. Das grosse

300 Netz ist massiv verdickt und weist auf der Innenseite eine echogene knotige Auflagerung auf. Die

301 Untersuchung erfolgte mit einem 5.0 MHz-Linearschallkopf von der rechten ventralen Bauchwand

302 aus. 1 Ventrale Bauchwand, 2 Flüssigkeit ausserhalb des Netzbeutels, 3 Flüssigkeit innerhalb des

303 Netzbeutels, 4 Stark verdicktes grosses Netz, 5 Echogene knotige Auflagerung auf der Innenseite des

304 grossen Netzes, Cr Cranial, Cd Caudal.

305

306 Abbildung 3: Kleine (A) und grosse (B) mit Flüssigkeit gefüllte Blasen auf dem grossen Netz einer

307 10-jährigen Braunviehkuh mit Mesotheliose.

309 Abbildung 4: Histologisches Bild. Biphasische Neoplasie bestehend aus einer epithelialen (Pfeile)

310 und einer mesenchymalen Komponente (Stern). Die Epithelien zeigen deutliche Zilien, die

311 mesenchymalen Zellen Anisozytose und Anisokaryose. HE-Färbung.

313 Abbildung 5: Immunhistologisches Bild. Epitheliale Komponente (Pfeile) mit stark positivem Signal 314 für epitheliale Intermediärfilamente; Pan-Zytokeratin-Marker (Lu 5).

316 Abbildung 6: Elektronenmikroskopisches Bild einer Zellgrenze zwischen zwei epithelialen

317 neoplastischen Zellen mit ausgeprägten, unterschiedlich langen und unregelmässigen Desmosomen-

318 ähnlichen Strukturen (Pfeile). Die Zellkerne (Sterne) sind links oben und rechts unten angeschnitten. 